\title{
INFLUÊNCIA DA ADIÇÃO DE FONTES MARINHAS RICAS EM PUFAs NA DIETA SOBRE A COMPOSIÇÃO LIPÍDICA E PERCENTUAIS DE INCORPORAÇÃO DE PUFAs N-3 NA GEMA DO OVO
}

\section{P.R. Carvalho' ${ }^{\text {, M.C.G. Pita }}{ }^{2}$, E. Piber Neto², C.X. Mendonça Junior ${ }^{2}$}

${ }^{1}$ Pólo Apta Regional do Centro-Oeste, Unidade de Pesquisa de Bauru, Av. Rodrigues Alves, 40-40, CEP 17063080, Bauru, SP, Brasil. E-mail: p_reiscar@apta.sp.gov.br

\section{RESUMO}

\begin{abstract}
A presente pesquisa foi conduzidautilizando-se 288 galinhas poedeiras dalinhagem Hisex White com 32 semanas de idade, pelo período de 10 semanas, com o objetivo de estudar o enriquecimento da gema do ovo em ácidos graxos, a partir de rações suplementadas com óleo de peixe (OP) ou alga marinha (AM) em cinconíveis deDHA $(120,180,240,300$ e360 mg/100 g dieta). Foi aplicado o modelo fatorial $2 \times 5$, inteiramente casualizado, com três repetições de oito aves por tratamento, de modo a constituir os grupos:OP120,OP180,OP240,OP300,OP360, AM120, AM180, AM240, AM300e AM360. Um grupo controle submetido à ração basal de milho e soja (CON) e outro contendo $420 \mathrm{mg}$ de DHA/ $100 \mathrm{~g}$ dieta (AM420) acrescido de AM, foram também utilizados. Quanto aos teores de DHA na gema do ovo de aves suplementadas com OP, foi observado aumento significativo de 22,64 mg/gema (CON), para 187,91 mg/gema no grupo OP360. Os PUFAs n-3 apresentaram acréscimo significativo no contraste entre CON (62,16 mg/gema) e OP360 (218,62 mg/gema). Para a fonte AM, as médias de DHA também mostraram linearidade $\left(Y=0,23 X+1,27, \mathrm{R}^{2}=0,86\right)$, oscilando entre $22,64 \mathrm{mg} /$ gema (CON) e 149,75 mg/gema (AM420), enquanto que o total de PUFAs n-3 oscilou de 104,18 mg/gema (AM120) a 175,32 mg/gema (AM420). O percentual de incorporação de DHA na gema decresceu linearmente com o aumento dos níveis de DHA na ração suplementada com OP e AM, de 85,11\% (OP120) e 65,28\% (AM120) para 49,45\% (OP360) e 34,06\% (AM420). Melhora significativa ( $<<0,05)$ foi consignada na relação n-6/n-3, que variou de 17,50 no grupo CON para 3,72 e 6,36 nos tratamentos OP360 e AM420, respectivamente.
\end{abstract}

PALAVRAS-CHAVE: Incorporação de PUFAs n-3, ovos de galinha, DHA, óleo de peixe, alga marinha.

\section{ABSTRACT}

INFLUENCE OF PUFA-RICH DIETARY SUPPLEMENTS FROM MARINE SOURCES IN THE FEEDOF LAYINGHENSON LIPIDCOMPOSITION ANDTHE PERCENTAGEOFINCORPORATION OF N-3 PUFAS IN EGG YOLK. This experiment was conducted using 28832-week-old Hisex White laying hens for a period of 10 weeks, with the objective of studying the fatty acid enrichment of the eggyolk of hens fed diets supplemented with fishoil(OP) or marinealgae(AM) to providefivelevels of DHA (120, 180, 240, 300 and $360 \mathrm{mg} / 100 \mathrm{~g}$ diet) for each source. A 2 × 5 completely randomized factorial design with three replicates of 8 birds per treatment was applied in order to have the following groups: OP120, OP180, OP240, OP300, OP360, AM120, AM180, AM240, AM300 and AM360. A control group submitted to a corn/soy basal diet $(\mathrm{CON})$ and another one supplemented with $\mathrm{AM}$ at the level of $420 \mathrm{mg}$ of DHA/100 $\mathrm{g}$ diet (AM420) were also used. The amounts of DHA in the egg yolk in birds fed OP diets were significantly increased from $22.64 \mathrm{mg} / \mathrm{egg}$ yolk (CON) to $187.91 \mathrm{mg} /$ egg yolk (OP360). The egg-yolk n-3 PUFAs of the control group $(62.16 \mathrm{mg})$ increased significantly as compared to the OP360 group (218.62 mg/yolk). For the AM source the DHA means were also linear $\left(\mathrm{Y}=0.23 \mathrm{X}+1.27, \mathrm{R}^{2}=0.86\right)$, ranging from $22.64 \mathrm{mg} /$ yolk $(\mathrm{CON})$ to $149.75 \mathrm{mg} /$ yolk (AM420), while the n-3 PUFAs ranged from $104.18 \mathrm{mg} /$ yolk (AM120) to $175.32 \mathrm{mg} /$ yolk (AM420). The percentage of DHA incorporation into the egg yolk decreased linearly as the DHA levels increased in the diet. Thus, for the OP and AM sources, mean values of $85.11 \%$ (OP120) and $65.28 \%$ (AM120)

${ }^{2}$ Universidade de São Paulo, Faculdade de Medicina Veterinária e Zootecnia, Departamento de Clínica Médica, São Paulo, SP, Brasil. 
decreased to $49.45 \%$ (OP360) and 34.06\% (AM420). Significant improvement $(\mathrm{P}<0.05)$ was found in the ratio $n-6 / n-3$, ranging from $17.50(\mathrm{CON})$ to $3.72(\mathrm{OP} 320)$ and 6.36 (AM420).

KEY WORDS: Incorporation of n-3 PUFAs, hens' eggs, DHA, fish oil, marine algae.

\section{INTRODUÇÃO}

A macroestrutura do ovo consiste em média de 9 a $12 \%$ decasca, $60 \%$ dealbúmene de 30 a 33\% de gema (Stadelman, 1995). A gema tem em média 33\% de lipídeos (COTTERIL; GeIGER, 1977), com a seguinte composição: $63,3 \%$ de triacilgliceróis, 29,7\% de fosfolipídios (fosfatidilcolina: $73 \%$; fosfatidiletanolamina: $15 \%$ ) e $5,2 \%$ de colesterol total (LESKANICH; Noble,1997).

$\mathrm{Na}$ atualidade, as linhagens selecionadas de aves poedeiras comerciais (Gallus gallus domesticus L.) produzem, em média, acima de 328 ovos por ciclo de postura (SCHEUERMANN; BELLAVER, 1995) e peso médio do ovo ao redor de 60 gramas (Li-ChAn etal., 1995). Tornase necessário o balanceamento das dietas visando o equilíbrio em proteínas, carboidratos, lipídeos, minerais e vitaminas em função do aumento do aporte de nutrientes em macro e micronutrientes essenciais à manutenção da poedeira e formação do ovo (S TADELMAN; PRATT, 1989). Considerando o ovo célula vitalícia que irá gerar o embrião, deve conter todos os nutrientes necessáriosao desenvolvimento donovoser(GRIMINGER, 1986; EтCHEs, 1996). Do ponto devista nutricional, o ovo é considerado alvo ideal para modificação da dieta humana e animal conduzindo ao desenvolvimento de alimento com propriedades funcionais ou nutracêuticas. SuRAI; SPARKS (2001) e ZEIDLER (1998) consideraram que os benefícios da melhora da qualidade do ovo com aumento da concentração de ácidos graxos poliinsaturados ômega-3 (PUFAs n-3), vitamina $\mathrm{E}$, carotenóides e selênio refletem os resultados das pesquisas visando enriquecer ovos. Agema possui alto teor de gorduras passíveis de alteração da composição química de seus ácidos graxos (YU; SIM, 1987; GRIFFIN, 1992; SIM , 2000). O conhecimento destas propriedades é importante ao permitir adoção de estratégicas visando modificar as características de seus ácidos graxos. Alguns destes ácidos graxos, denominados de PUFAs de cadeia longa da série n-3, eicosapentaenóico (EPA, $\mathrm{C}_{20: 5 \mathrm{n}-3}$ ) e docosahexaenóico (DHA), $\mathrm{C}_{22: 6 \mathrm{n}-3}$ ), participam como constituintes de membranas biológicas e organelas celulares, exercendo importantes funções bioquímicas em nível molecu-lar como sinalizadores célula a célula e precursores de substâncias com propriedades de reguladores hormonais autócrina e parácrina (PhettePlace; WATKINS, 1989). Do EPA originam-seas prostaglandinas, tromboxanoseleucotrienos das séries 3 e5. Ao aumento deDHA eEPA na dieta esta ligado ao desenvolvimento cerebral normal, à prevenção de inúmeras patologias entre as quais as doenças cardiovasculares (DCV), diabetes, lupus, psoríase, câncer (NETTLETON, 1995) e distúrbio visual (NEURINGER et al., 1984).

A fortificação de ovos tem sido usada estrategicamente para suprir a carência da indústria farmacêutica e de alimentos nas formulações de produtos nutracêuticos ou funcionais, a partir de fonte animal, para obtenção de lecitina e óleo de gema enriquecidos nos PUFAs n-3, EPA e DHA, entre as múltiplas finalidades, a de atender as exigências do DRA (NATIONAL ReseARCH Council, 1989) nas formulações de infantis lactentes e de gestantes (SIMOPOULOS et al.,1994). Emrecentes pesquisas realizadas, SPARKIS; Surai (2001) utilizaram óleo de peixe (OP) na dieta e obtiveram ovos fortificados com 200 mg PUFAs n-3/ ovo; HARGIs et al. (1991), com o uso da fonte de OP, obtiveram acima de $180 \mathrm{mg}$ /ovo de EPA + DHA; ABRIL; B ARCLAY (1998) reportaram o uso de microalgas marinha (MA) e os ovos enriquecidos apresentaram médias acima de 172,8 mg de PUFAs n-3 e Herber; VAN ELSWYK (1996) suplementaram a ração de poedeiras com MA e ovos enriquecidos mostraram médias acima de 9,5 mg/g de gema de PUFAs n-3. Objetivou-se nesta pesquisa estudar a influência da suplementação à ração de teores crescentes de óleo de peixe e de alga marinha, sobre o perfil de PUFAs n-3 e os percentuais de incorporação na gema do ovo de galinhas poedeiras submetidas à dieta basal de milho e soja.

\section{MATERIAL E MÉTODOS}

A pesquisa foi conduzida no biotério experimental de aves do Departamento de Clínica Médica da Faculdade de Medicina Veterinária e Zootecnia da Universidade de São Paulo. No presente estudo foi utilizado o delineamento inteiramente casualizado, empregando-se 288 galinhas poedeiras da linhagem comercial Hisex White com 32 semanas idade, distribuídas em 12 tratamentos, com três repetições de oito aves, alojadas em gaiolas de $0,45 \mathrm{~m} \times 0,25 \mathrm{~m}$ x 0,45 m, sendo duas aves por gaiola. A ração foi fornecida ad libitum em comedouro tipo calha e a água em bebedouro tiponipple. As aves receberam 16 horas diárias de luz.

\section{Rações experimentais}

As rações experimentais dos 12 tratamentos, fornecidas às galinhas poedeiras, isocalóricas e 
isoprotéicas, foram formuladas de acordo com os requerimentos estabelecidos pelo NATIONAL RESEARCH Council (1994). O grupo controle (Tabela 1) foi composto de dieta basal de milho efarelo de soja, enquanto que nos demais tratamentos as rações foram suplementadas com óleo de salmão (OP) ou alga (AM). Os tratamentos de dois a seis tiveram adição de níveis crescentes de DHA de óleo de salmão (Salmo salar) nas concentrações de $120 \mathrm{mg}(0,80 \%$ OP); $180 \mathrm{mg}(1,20 \%$ OP); $240 \mathrm{mg}$ (1,60\% OP); $300 \mathrm{mg}$ $(2,00 \%$ OP) e 360 mg DHA/ 100 g ração $(2,40 \%$ OP), enquanto que os de sete a doze continham percentuais crescentes de alga marinha (Schizochytrium sp.) de $120 \mathrm{mg}$ (0,50\% AM); $180 \mathrm{mg}$ (0,75\%); $240 \mathrm{mg}(1,00 \%$ AM); $300 \mathrm{mg}$ (1,25\% AM); $360 \mathrm{mg}$ (1,50\% AM) e 420 mg DHA/ $100 \mathrm{~g}$ de ração (1,75\% AM). Ao OPe AM foram adicionados 200 ppm do antioxidante ButilHidroxi-Tolueno (BHT).

As composições do total de PUFAs da sérien-3 nas fontes utilizadas deóleo de peixe (OP) ealga marinha (AM) foram, respectivamente: $\mathrm{EPA}\left(\mathrm{C}_{20: 5 \mathrm{n}-3}\right)-11,44 \% \mathrm{e}$ $2,74 \%$; DPA $\left(C_{22: 5 n-3}\right)-4,43 \%$ e $17,08 \%$; DHA $\left(C_{22: 6 n-3}\right)$ $-15,03 \%$ e $42,38 \%$. As relações do total de n- $6 / n-3$ nas fontes OPe AM foram de 0,24 e 0,05, respectivamente. O extrato etéreo da alga marinha foi $56,20 \%$. A alga marinha e óleo de milho continham antioxidante adicionado pelo fornecedor.

\section{Análise dos ácidos graxos da gema do ovo}

Na oitava semana experimental, foram colhidos quatro ovos por repetição. As gemas foram separadas e obtido o peso por unidade. A seguir, foram homogeneizadas a fim de se obter uma amostra por repetição (formada por um pool de quatro gemas), constituindo-se três amostras por tratamento. As análises foram realizadas a partir de um grama de gema fresca e crua de cada amostra segundo a metodologia descrita por Bligh; Dyer (1959), FolCh et al. (1957), modificado por NieLSEN (1998) eAsSOciATION OFOfFICIAL Agricultural Chemist (1970). Utilizou-se a técnica descrita por HARTMAN;LAGO (1973) para saponificação do extrato lipídico e obtenção dos ésteres de ácidos graxos das amostras. A seguir, a amostra foi solubilizada com hexano e procedeu-se a injeção de 1 (um) $\mu \mathrm{L}$ da solução para determinação do perfil de metil-ésteres de ácidos graxos através do uso da técnica de cromatografia gasosa.

Para a avaliação do perfil de ácidos graxos da $\mathrm{AM}$, dos óleos de peixe e milho, das rações e das gemas (Tabelas 1, 2 e 3) utilizou-se a técnica de cromatografia gasosa com o uso de cromatógrafo da marca Varian modelo CP 3800 equipado com detector de ionização de chama e acoplado ao sistema "Workstation Star Chromatography". Empregou-se coluna capilar de sílica fundida CP-WAX
52CB (Chrompack) com $30 \mathrm{~m}$ de comprimento, 0,25 $\mathrm{mm}$ de diâmetro e $0,25 \mu \mathrm{m}$ de polietilenoglicol. As condições de operação foram: injeção "split" , razão 50:1, temperatura da coluna: $150^{\circ} \mathrm{C}$ durante 15 minutos, programada até $210^{\circ} \mathrm{C}$ em uma razão de $3^{\circ} \mathrm{C}$ por minuto; gás de arraste: nitrogênio com uma vazão de 1,5 mL por minuto; gás "make-up": nitrogênio $30 \mathrm{~mL}$ por minuto; temperatura do injetor: $250^{\circ} \mathrm{C}$; temperatura do detector: $280^{\circ} \mathrm{C}$. Foram utilizados padrões externos contendo o perfil de metil-ésteres de ácidos graxos 189-19 da Supelco ${ }^{\circledR}$. Foi utilizado o padrão interno de etil-éster de ácido docosahexaenóico da Sigma ${ }^{\circledR}$ cis - 4.7.10.13.16.19a 99\% (D-2661, $10 \mathrm{mg}$ ).

\section{Análise estatística}

Na avaliação estatística dos resultados foi utilizado modelo fatorial $2 \times 5$ [fontes de ácidos graxos poliinsaturados: óleo de peixe (OP) e alga marinha (AM)] e níveis de ácido docosahexaenóico (DHA) nas dietas contendo OP e AM: 120; 180; 240; 300 e $360 \mathrm{mg} / 100 \mathrm{~g}$ ) com três repetições por tratamento em delineamento inteiramente casualizado aplicando-se os procedimentos descritos porSNEDECOR; Cochran (1967). Com o objetivo de comparar os tratamentos previamente mencionados com um grupo controle de aves alimentadas com ração basal de milho e soja e outro constituído de galinhas submetidas à dieta contendo AM em teor de $420 \mathrm{mg}$ de DHA/100 g de ração, foi elaborada a análise de variância envolvendo o total de doze tratamentos. O teste de Tukey foi aplicado para analisar a diferença entre médias. A análise estatística foi realizada mediante o uso do software "Statistical Analysis System" (SAS, 1994) adotando o nível de 5\% de significância.

\section{RESULTADOS}

A adição de teores crescentes de fontes suplementares de DHA à ração das poedeiras (Tabelas 1 e 2) influenciou as médias referentes aos totais de ácidos graxos saturados (\% SAT), monoinsaturados (\% MUFAs), poliinsaturados (\% PUFAs), \% PUFAs n-3 e as relações $n-6 / n-3$, poliinsaturado:saturado (P/S), nos tratamentos, fontes eníveis deOPeAMestudados de acordo com o peso das gemas, lipídios totais (\%) e gordura da gema (Tabelas 3 a 6).

As maiores concentrações em ordem crescente de SAT na gema do ovo foram mirístico < esteárico < palmítico. Dentre estes, apenas oácido esteárico $\left(C_{18: 0}\right)$ não apresentou diferenças significativas entre tratamentos pela análise de variância. 


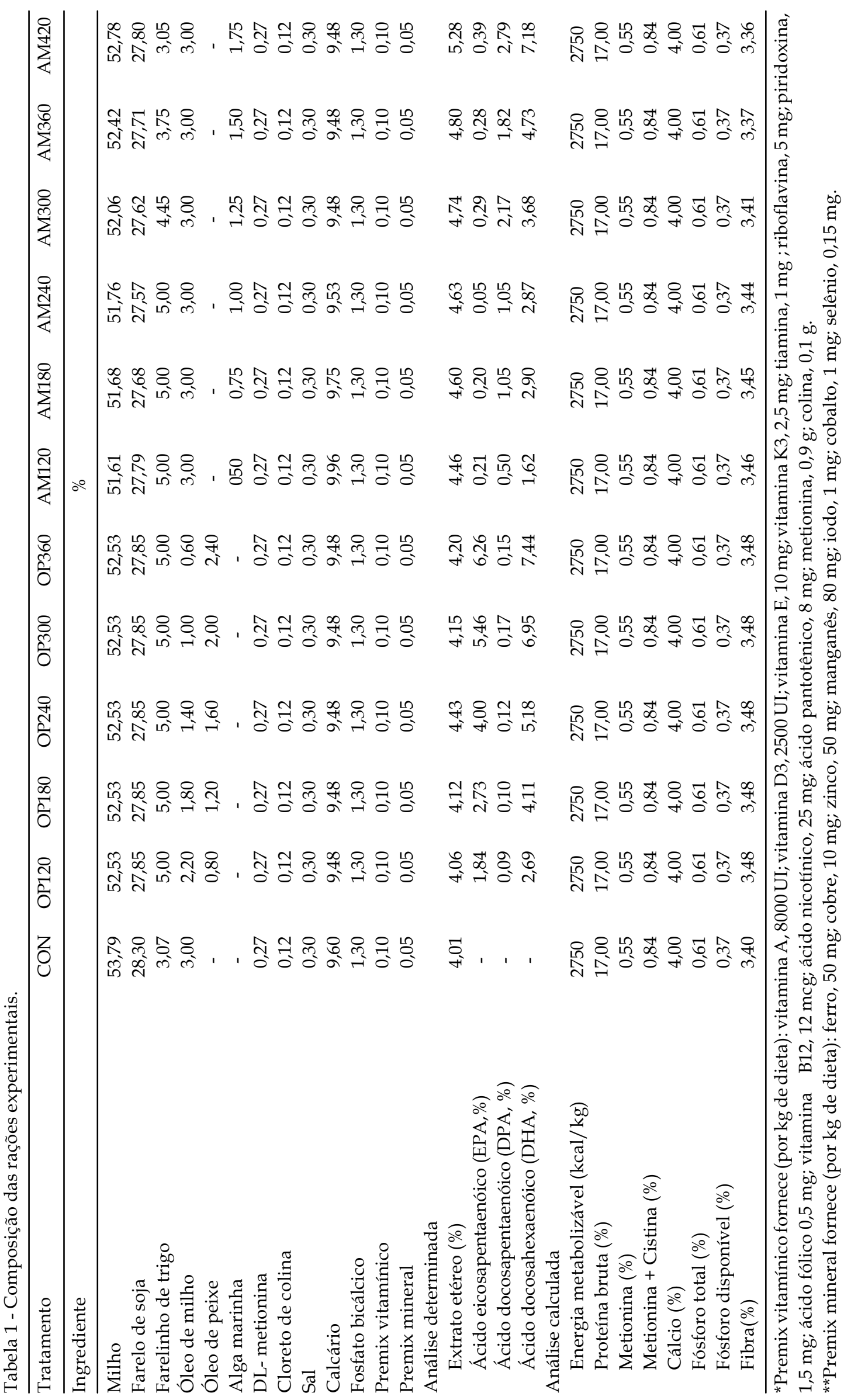


Influência da adição de fontes marinhas ricas em PUFAs na dieta sobre a composição lipídica e percentuais de incorporação de PUFAs n-3 na gema do ovo.

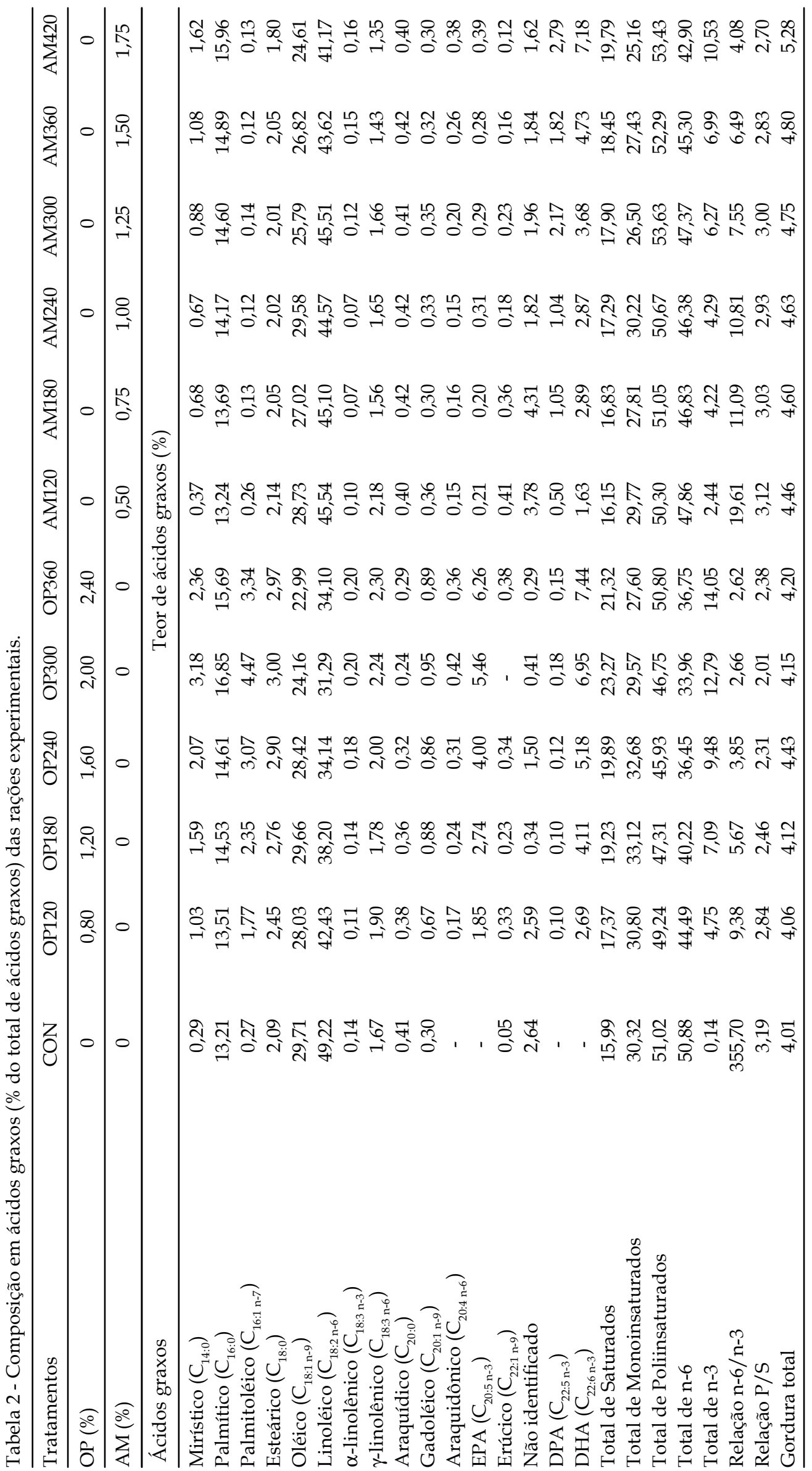




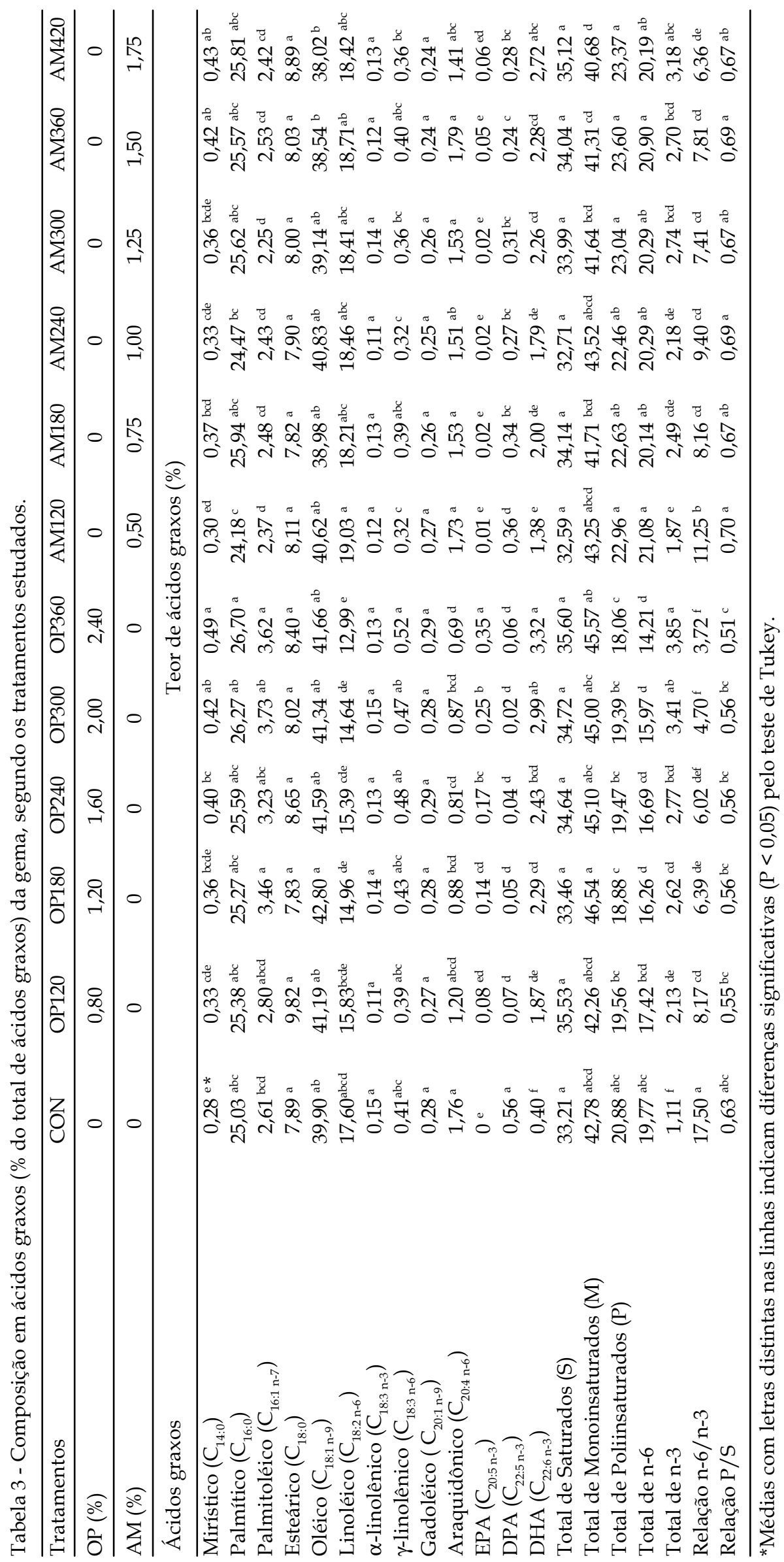


Os teores totais de SAT (\%) na gema não foram influenciados $(\mathrm{P}>0,05)$ pela adição de níveis crescentes de DHA. Por sua vez, a média significativamente mais elevada foi obtida com a utilização da fonte de OP $(34,79 \%)$ quando comparada àquela assinalada com o uso de AM (33,49\%) (Tabela 4).

Os \% MUFAs representaram o maior teor de ácido graxo da gema, sendo o ácido oléico responsável por esta expressiva diferença. As concentrações de MUFAs em ordem crescente foram gadoléico < palmitoléico < oléico (Tabela 3).

A influência das fontes foi significativa sobre o teor de MUFAs na gema, sendo a média 45,29\% (OP) diferente da menor média de $42,28 \%$ apresentada pela fonte de AM.

O total de PUFAs decresceu significativamente de $20,88 \%$ (CON) para 18,06\% (OP360) nas dietas com OP. Médias maiores variando de 22,63\% (AM180) a 23,60\% (AM360) não diferentes do CON, foram obtidas nas dietas suplementadas com AM sendo representadas majoritariamente pelo acido linoléico (AL), o terceiro maior ácido da gema (Tabela 3).

Em relação à influência das fontes, a média significativamente mais elevada foi notada com a utilização da fonte de AM (22,93\%) quando comparada àquela assinalada com o uso de OP (19,07\%) (Tabela 4).

Dentre os quatro PUFAs n-3 presentes na gema e estudados nesta pesquisa, a penas oácido $\alpha$-linolênico não apresentou diferenças julgadas significativas entre tratamentos pela análise de variância (Tabela 3).
As médias de DHA (\%) na gema do ovo mostraram acréscimos significativos na medida em que níveis crescentes de DHA da fonte OP foram suplementados à ração das poedeiras. As médias de 3,32\% (OP360) diferiram das médias dos tratamentos suplementados com OP, exceto da média de 2,99\% (OP300) na gema do ovo. Ainda, com níveis crescentes de DHA nos tratamentos suplementados com AM na ração, os valores médios entre1,38\% (AM120) a 2,72\% (AM420) de DHA na gema do ovo também diferiram da média de $0,40 \%$ (CON). Observou-senas médias (\%) de DHA na gema do ovo resposta linear em relação aos níveis crescentes de DHA das fontes de OP e AM nos tratamentos estudados, sendo expresso pela regressão linear $Y=0,23 X+1,27\left(R^{2}=0,86\right)$ para a fonte de AM na dieta (Tabela 3 ).

As fontes e níveis de DHA na dieta mostraram efeito significativo sobre os teores de DHA na gema, sendo mais efetiva a fonte de OP na ração (Tabela 4).

A média do total dePUFAsn-3(\%) de3,85\% (OP360) diferiu das médias dos tratamentos contendo níveis crescentes de DHA na ração da fonte de OP, exceto da média de 3,41\% (OP300) na gema do ovo. As fontes definitivamente influenciaram o total dePUFAsn-3 na gema dos ovos. A maior média de 2,95\% (OP) foi diferente $(\mathrm{P}<0,05)$ da média de $2,39 \%$ de PUFAs $n-3$ (AM) na gema do ovo. Entre níveis, a menor média de $2,00 \%$ (120 mg de DHA) diferiu em relaçãoàs maiores médias de 3,07\% e 3,27\% de PUFAs n-3 (300 mg e 360 mg DHA, respectivamente) na gema (Tabela 3 e 4 ).

Tabela 4 - Composição em ácidos graxos da gema (\% do total de ácidos graxos), segundo as fontes e níveis estudados.

\begin{tabular}{|c|c|c|c|c|c|c|c|}
\hline \multirow[t]{2}{*}{ Ácidos graxos } & \multicolumn{2}{|c|}{ Fontes } & \multicolumn{5}{|c|}{ Níveis } \\
\hline & OP & $\mathrm{AM}$ & 120 & 180 & 240 & 300 & 360 \\
\hline Mirístico $\left(\mathrm{C}_{14: 0}\right)$ & $0,40^{a} *$ & $0,35^{\mathrm{b}}$ & $0,31^{\mathrm{C}}$ & $0,36^{\mathrm{BC}}$ & $0,36^{\mathrm{BC}}$ & 0,39 Ав & $0,46^{\mathrm{A}}$ \\
\hline Palmítico $\left(\mathrm{C}_{16: 0}\right)$ & $25,84^{\mathrm{a}}$ & $21,15^{b}$ & $24,78^{\text {в }}$ & $25,61^{\mathrm{AB}}$ & 25,03 АВ & $25,94 \mathrm{AB}$ & $26,13^{\mathrm{A}}$ \\
\hline Palmitoléico $\left(\mathrm{C}_{16: 1 \mathrm{n}-7}\right)$ & $3,29^{a}$ & $2,41^{\mathrm{b}}$ & $2,58^{\mathrm{A}}$ & $2,97^{\mathrm{A}}$ & 2,82 A & $2,81^{\mathrm{A}}$ & $3,07^{\mathrm{A}}$ \\
\hline Esteárico $\left(\mathrm{C}_{18: 0}\right)$ & $8,54^{\text {a }}$ & $7,97^{a}$ & $8,96^{\mathrm{A}}$ & $7,82^{\mathrm{A}}$ & $8,28^{\mathrm{A}}$ & $8,01^{\mathrm{A}}$ & $8,22^{\mathrm{A}}$ \\
\hline Oléico $\left(C_{18: 1 \mathrm{n}-9}\right)$ & $41,71^{\text {a }}$ & $39,62^{b}$ & $40,90^{\mathrm{A}}$ & 40,89 A & $41,20^{\mathrm{A}}$ & $40,24^{\mathrm{A}}$ & $40,09^{\mathrm{A}}$ \\
\hline Linoléico $\left(\mathrm{C}_{18: 2 \mathrm{n}-6}\right)$ & $14,76^{\mathrm{b}}$ & $18,56^{\text {a }}$ & $17,43^{\mathrm{A}}$ & $16,58^{\mathrm{A}}$ & $16,92^{\mathrm{A}}$ & $16,52^{\mathrm{A}}$ & $15,85^{\mathrm{A}}$ \\
\hline$\alpha$-linolênico $\left(\mathrm{C}_{18: 3 \mathrm{n}-3}\right)$ & $0,12^{\text {a }}$ & $0,12^{\text {a }}$ & $0,12^{\text {в }}$ & 0,13 АВ & $0,12^{\text {в }}$ & $0,14^{\mathrm{A}}$ & $0,12 \mathrm{AB}$ \\
\hline$\gamma$-linolênico $\left(C_{18: 3 n-6}\right)$ & $0,45^{\mathrm{a}}$ & $0,35^{b}$ & $0,36^{\mathrm{A}}$ & $0,40^{\mathrm{A}}$ & $0,40^{\mathrm{A}}$ & $0,41^{\mathrm{A}}$ & $0,46^{\mathrm{A}}$ \\
\hline Gadoléico $\left(\mathrm{C}_{20: 1 \mathrm{n}-9}\right)$ & $0,28^{a}$ & $0,25^{b}$ & $0,26^{\mathrm{A}}$ & $0,27^{\mathrm{A}}$ & $0,27^{\mathrm{A}}$ & $0,27^{\mathrm{A}}$ & $0,26^{\mathrm{A}}$ \\
\hline Araquidônico $\left(\mathrm{C}_{20: 4 \mathrm{n}-6}\right)$ & $0,89^{b}$ & $1,61^{\mathrm{a}}$ & $1,46^{\mathrm{A}}$ & $1,21^{\mathrm{A}}$ & $1,16^{\mathrm{A}}$ & $1,20^{\mathrm{A}}$ & $1,24^{\mathrm{A}}$ \\
\hline $\operatorname{EPA}\left(\mathrm{C}_{20: 5 \mathrm{n}-3}\right)$ & $0,20^{a}$ & $0,02^{b}$ & $0,04^{\mathrm{A}}$ & $0,08^{\mathrm{A}}$ & 0,09 A & $0,14^{\mathrm{A}}$ & $0,20^{\mathrm{A}}$ \\
\hline $\operatorname{DPA}\left(C_{22: 5 n-3}\right)$ & $0,04^{b}$ & $0,30^{a}$ & $0,21^{\mathrm{A}}$ & $0,19^{\mathrm{A}}$ & $0,15^{\mathrm{A}}$ & $0,17^{\mathrm{A}}$ & $0,15^{\mathrm{A}}$ \\
\hline DHA $\left(C_{22: 6 n-3}\right)$ & $2,57^{\mathrm{a}}$ & $1,94^{\mathrm{b}}$ & $1,63^{\text {в }}$ & $2,14 \mathrm{AB}$ & $2,10 \mathrm{AB}$ & $2,63^{\mathrm{A}}$ & $2,80^{\mathrm{A}}$ \\
\hline Total de Saturados & 34,79 a & $33,49^{b}$ & $34,06^{\mathrm{A}}$ & $33,80^{\mathrm{A}}$ & $33,67^{\mathrm{A}}$ & $34,35^{\mathrm{A}}$ & $34,81^{\mathrm{A}}$ \\
\hline Total de Monoinsaturados & $45,29^{\text {a }}$ & $42,28^{b}$ & $43,75^{\mathrm{A}}$ & $44,13^{\mathrm{A}}$ & $44,31^{\mathrm{A}}$ & $43,32^{\mathrm{A}}$ & $43,44^{\mathrm{A}}$ \\
\hline Total de Poliinsaturados & $19,07^{b}$ & $22,93^{\text {a }}$ & $21,25^{\mathrm{A}}$ & $20,75^{\text {A }}$ & $20,96^{\mathrm{A}}$ & $21,21^{\mathrm{A}}$ & $20,83^{\mathrm{A}}$ \\
\hline Total de n-6 & $16,11^{\mathrm{b}}$ & $20,53^{\text {a }}$ & $19,25^{\mathrm{A}}$ & $18,19^{\mathrm{A}}$ & $18,49^{\mathrm{A}}$ & $18,13^{\mathrm{A}}$ & $17,55^{\mathrm{A}}$ \\
\hline Total de $n-3$ & $2,95^{\mathrm{a}}$ & $2,39^{\mathrm{b}}$ & $2,00^{\mathrm{C}}$ & $2,55^{\mathrm{ABC}}$ & $2,47^{\mathrm{BC}}$ & $3,07{ }^{\mathrm{AB}}$ & $3,27^{\mathrm{A}}$ \\
\hline Relação n-6/n-3 & $5,79^{b}$ & $8,80^{a}$ & $9,71^{\mathrm{A}}$ & 7,27 АВ & $7,71^{\mathrm{AB}}$ & $6,05^{\text {в }}$ & $5,76^{\text {в }}$ \\
\hline Relação P/S & $0,54^{\mathrm{b}}$ & $0,68^{a}$ & $0,62^{\mathrm{A}}$ & $0,61^{\mathrm{A}}$ & $0,62^{\mathrm{A}}$ & $0,61^{\mathrm{A}}$ & $0,59^{\mathrm{A}}$ \\
\hline
\end{tabular}

*Médias com letras distintas nas linhas indicam diferenças significativas $(\mathrm{P}<0,05)$ pelo teste de Tukey. 
As médias do total de EPA (mg/gema) nos tratamentos aumentaram $(\mathrm{P}<0,05)$ com o acréscimo dos teores de OP na ração das galinhas poedeiras. Um Aumento linear significativo de 4,59 mg (OP120) a 19,70 mg EPA/gema foi obtido com acréscimo de fonte animal deOP à dieta. O oposto foi observadonos tratamentos com fonte de origem de AM (Tabela 5).

As médias (mg/gema) de DHA nos tratamentos foram significativamente influenciadas quando níveis crescentes de DHA da fonte OP e AM foram suplementados à ração das poedeiras. As médias de 187,91 mg DHA/gema (OP360) diferiram significativamente das médias dos demais tratamentos contendo a fonte OP na dieta, exceto da média de158,61 mg/ gema (OP300). As médias de DHA na gema do ovo com níveis crescentes de DHA da fonte AM na ração mostraram acréscimos significativos, variando de 77,02 mg (AM120), a 149,75 mg DHA/gema do ovo (AM420) (Tabela 5).

Entre fontes, a maior média de 142,33 mg (OP) diferiu da média de 107,84 mg DHA/gema (AM). Entre níveis, as maiores médias de 139,15 mg (300 mg DHA) e 157,72 mg DHA/gema (360 mg DHA) diferiram da menor de 90,90 mg/gema de DHA (120 mg DHA) (Tabela 6).

Na soma do total dePUFAsn-3 (ALA + EPA + DPA + DHA), as médias de 13,27 mg PUFAs/g e 218,22 mg PUFAsn-3/gema diferiram em relação as observadas nos dos demais tratamentos, exceto para as médias de OP300 e AM420 (Tabela 5).

Entre fontes, o maior valor de 9,28 mg (OP) não mostrou diferença da média de 7,97 mg PUFAs n-3/ g gema e o contrário foi verificado com as médias de 163,29 (OP) e 132,92 (AM) que, por influencia dos níveis de DHA na dieta, mostraram-se diferentes (Tabela 6).

As maiores de incorporação de DHA de 85,11\% (OP120) e 70,10\% (OP180) foram obtidos com a fonte de OP em menores níveis na dieta. Inversamente, consumos maiores de 246,42(OP240) e de 241,82 mg/ ave/dia (AM240) determinaram incorporações significativamente decrescentes, respectivamente, com médias de 49,45\% e 40,51\% (Tabela 5).

Entrefontes, as médias de consumo de 245,98(OP) e 239,68 mg/ave/dia (AM), consideradas não diferentes, determinaram incorporações significativas sendo a maior média de $62,44 \%$ (OP) diferente de 49,45\% (AM) (Tabela 6).

\section{DISCUSSÃO}

A avaliação da composição dos lipídes da gema de ovos, no presente estudo, permitiu observar a influência marcante das dietas sobre as alterações ocorridas na composição final em ácidos graxos na gordura da 
Tabela 6 - Médias de PUFAs n-3 (mg e g/gema) e totais de PUFAs n-3 (mg/gema), consumo de DHA (mg/ave/dia), incorporação (\%), peso da gema (g), lipídeos totais (\%) e gordura da gema (g) de lipídeos totais médios da gema, segundo as fontes e níveis estudados.

\begin{tabular}{|c|c|c|c|c|c|c|c|}
\hline \multirow[t]{2}{*}{ Ácidos graxos } & \multicolumn{2}{|c|}{ Fontes } & \multicolumn{5}{|c|}{ Níveis } \\
\hline & $\mathrm{OP}$ & $\mathrm{AM}$ & 120 & 180 & 240 & 300 & 360 \\
\hline & \multicolumn{7}{|c|}{ Teores médios de PUFAs n-3 na gema } \\
\hline EPA (mg/gema) & $11,08^{a} *$ & $1,22^{b}$ & $2,41^{\mathrm{A}}$ & $4,84^{\mathrm{A}}$ & $5,10^{\mathrm{A}}$ & $7,27^{\mathrm{A}}$ & $11,12^{\mathrm{A}}$ \\
\hline DPA (mg/gema) & $2,73^{b}$ & $16,94^{\text {a }}$ & $12,06^{\mathrm{A}}$ & $11,20^{\mathrm{A}}$ & $8,44^{\mathrm{A}}$ & $9,01^{\mathrm{A}}$ & $8,46^{\mathrm{A}}$ \\
\hline DHA (mg/gema) & $142,33^{\text {a }}$ & $107,84^{b}$ & $90,90^{\mathrm{B}}$ & $123,27 \mathrm{AB}$ & 114,39 АВ & $139,15^{\text {A }}$ & $157,72^{\mathrm{A}}$ \\
\hline ALA (mg/gema) & $7,13^{a}$ & $6,91^{a}$ & $6,47^{\mathrm{A}}$ & $7,60^{\mathrm{A}}$ & $6,37^{\mathrm{A}}$ & 7,69 A & $7,00^{\mathrm{A}}$ \\
\hline Total n-3 (mg/g gema) & $9,28^{\text {a }}$ & $7,97^{\text {a }}$ & $6,69^{A}$ & $8,64^{\mathrm{A}}$ & $8,33^{\mathrm{A}}$ & $10,26^{\mathrm{A}}$ & $9,21^{\mathrm{A}}$ \\
\hline Total n-3 (mg/gema) & 163,29 a & $132,92^{b}$ & $111,86^{\mathrm{C}}$ & $146,92 \mathrm{ABC}$ & $134,32^{\mathrm{BC}}$ & $163,13^{\mathrm{AB}}$ & $184,31^{\mathrm{A}}$ \\
\hline Consumo de DHA (mg/ave/dia) & $245,98^{a}$ & $239,68^{a}$ & $120,91^{z}$ & $179,80^{\mathrm{D}}$ & $244,11^{\mathrm{C}}$ & $303,58^{\text {в }}$ & $365,74^{\mathrm{A}}$ \\
\hline Incorporação de DHA (\%) & $62,44^{\text {a }}$ & $49,45^{\mathrm{b}}$ & $75,19^{\mathrm{A}}$ & 68,39 A & $46,78^{\text {в }}$ & $46,17^{\text {в }}$ & $43,19^{\text {в }}$ \\
\hline Peso da gema $(\mathrm{g})$ & $16,31^{\text {a }}$ & $16,71^{\mathrm{a}}$ & $16,75^{\mathrm{A}}$ & $17,05^{\mathrm{A}}$ & $16,17^{\mathrm{A}}$ & $15,87^{\mathrm{A}}$ & $16,70^{\mathrm{A}}$ \\
\hline Lipídeos totais (\%) & 33,77 a & $33,21^{a}$ & $33,28^{A}$ & $33,64^{\mathrm{A}}$ & $33,64^{\mathrm{A}}$ & $33,28^{A}$ & $33,62^{A}$ \\
\hline Gordura da gema $(\mathrm{g})$ & $5,51^{\mathrm{a}}$ & $5,54^{\mathrm{a}}$ & $5,57^{\mathrm{A}}$ & $5,73^{A}$ & $5,43^{\mathrm{A}}$ & $5,30^{\mathrm{A}}$ & $5,61^{\mathrm{A}}$ \\
\hline
\end{tabular}

*Médias com letras distintas nas linhas denotam diferenças significativas $(\mathrm{P}<0,05)$ pelo teste de Tukey.

gema por efeito dos tratamentos, das fontes e dos níveis de DHA na ração das poedeiras. Tais resultados estão de acordo com VAN ELSWYK (1997), BARCLAY et al. (1998), ABril et al. (2000) e GALOBART et al. (2002) ao reportarem a relação dizraxos da gema de ovos de galinhas poedeiras.

Os valores médios deSAT na gema permanceram ao redor de $33 \%$ nos tratamentos não diferindo do CON, concordando com GuARDiola et al. (1994) ao reportarem o mesmo percentual. Mesmo nas dietas suplementadas com fontes de PUFAs n-3, os valores totais médios de SAT de 34,79\% (OP) e de 33,49\% (AM) situaram-se ao redor de um terço (Tabela 4). Os maiores contribuintes para esse total no grupo controle foram as médias de $25,03 \%$ de ácido palmítico e 7,89\% de esteárico (Tabela 3), o que também foi observado por JIANG; SIM $(1993,1994)$. Tais resultados concordam ainda com as observações de CRUICKSHANK (1934) e FISHER;L EVEILLE (1957) ao reportarem que a presença nas dietas de elevados teores de ácidos graxos saturados decorrentes do acréscimo do teor de gordura saturada rica de fonte de óleo de palma ou gordura de carneiro à ração de poedeiras não influenciava a composição da gordura dos ovos, permanecendo similar àquela da dieta normal e, quando observada alguma variação, esta se mostrava mínima.

Os MUFAs em maior percentual na gema do ovo (Tabela 3) nesta pesquisa foram representados pelas médias de $42,80 \%$ para o oléico (OP180) e 3,73\% para o palmitoléico (OP300). De acordo com Noble et al. (1990), o padrão do ovo pós-postura envolve a maturação seqüencial da "ova" ou gema em intervalo de aproximadamente 24 horas. Os esforços metabóli- cos para sustentar o suprimento dos lipídeos para formação da gema são conseguidos por um sistema único de transporte e síntese altamente organizado (GRIFFIN et al., 1984; SIM, 1998). Com dietas baixas em gordura, a maioria dos ácidos graxos da gema é resultante da síntese "de novo" a partir de hidratos de carbono, enquanto que, com dietas ricas em gordura, grande parte é oriunda dos ácidos graxos dietéticos (NAber; Biggert, 1989). Diferentemente dos ácidos graxos saturados, a incorporação dos MUFAs à dieta pode aumentar seu nível na gema às expensas, principalmente, dos ácidos graxos mirístico, palmítico, palmitoléico e esteárico (DonALDSON, 1966; PANKeY; StADELMAN, 1969). Tipicamente, no ovo procedente de galinhas alimentadas com dieta convencional (milho/soja), o ácido oléico $\left(\mathrm{C}_{18: 1 \mathrm{n}-9}\right)$ é o ácido graxo em maior percentual (GUARDIOLAet al., 1994), concordando com o observado por HARGIS et al., (1991), VAN ELSWYK et al. $(1998,2000)$ e JIANG; SIM $(1993,1994)$.

Tais afirmações citadas pelos autores acima foram confirmadas nesta pesquisa quando as médias de $45,29 \%$ (OP) e de 42,28\% (AM) de MUFAs na gema evidenciaram influência significativa entre as fontes deOPe AMadicionadas à dieta (Tabela 4). Ainda, nas observações assinaladas por WATKINS; ELKIN (1992) e Pankey; Stadelman (1969), de que fonte de MUFAs adicionadas à dieta, a exemplo do óleo de oliva (teor elevado em $\left.\mathrm{C}_{18: 1 \mathrm{n}-9}\right)$, influenciariam mudanças significativas na composição em MUFAs da gema às expensas, principalmente, dos ácidos graxos mirístico, palmítico, palmitoléico e esteárico. Em relação às menores médias de MUFAs da fonte AM na dieta das poedeiras (Tabela 4), os resultados desta pesquisa corroboram os achados de Herber; vaN 
ELSWYK (1996) que, ao incrementar o teor de PUFAs n3 na gema de ovos com a incorporação de alga marinha à dieta de galinhas poedeiras, reportaram tendência ao decréscimo no decorrer das quatro semanas experimentais, e de JIANG; SIM (1994) eMori (2001) que, ao adicionarem teores crescentes de linhaça como fonte de PUFA n-3 à dieta de poedeiras, observaram redução dos MUFAs na gema do ovo.

As maiores médias de PUFA (\%) na gema foram obtidas com níveis crescentes de DHA de AMna dieta, sendo a fonte de OP mais efetiva em reduzir significativamente a média de 22,93\% (AM) para 19,07\% (OP) de PUFAs na gema (Tabelas 3 e 4). Esta influência pode ter sido exercida pela manutenção do teor de $3 \%$ de óleo de milho na ração nos tratamentos com teores crescentes de AM.

Os resultados da inclusão de PUFAS n-3 com 20 carbonos (EPA, $\left.\mathrm{C}_{20: 5 \mathrm{n}-3}\right)$ e 22 carbonos $\left(\mathrm{DHA}, \mathrm{C}_{22: 6 \mathrm{n}-3}\right)$ à ração de galinhas poedeiras de fonte OP mostraram reflexo direto na composição em ácidos graxos, com significativa fortificação em PUFAs n-3 na gema dos ovos, o que está de acordo com os dados apresentados em vários trabalhos realizados com diferentes fontes de PUFAs n-3 (óleos: peixe, linhaça, canola e ômega; sementes: linhaça e canola; farinhas: peixe e alga) e por diferentes autores de continentes distintos, dos quais podemos citar as pesquisas realizadas por JIANG et al. (1991), LESKANICH;NOBLE (1997), MARSHALl etal. (1994a), MARSHALl et al. (1994b) e SIM (2000). Entretanto, ao contrário dosaltosníveis deincorporaçãodosPUFAsn6 na gema dos ovos representados principalmente pelo ácido linoléico (CON-17,60\%), WHEELER et al. (1959) encontraram evidências de que, por exemplo, o ômega$3 \alpha$-linolênico e seus derivados EPA e DHA, com dieta rica contendo óleo de linhaça, era m inferiores a $15 \%$. CHENetal. (1965) observaram que, pela adição deóleode linhaça à ração, o linoléico apresentava-seincorporado emmaior teor nas frações triacilgliceróis comparadoaos fosfolipídios. Ao contrário, o EPA e DHA eram depositados exclusivamente nos fosfolipídios, preferencialmentenafraçãodafosfatidiletanolamina, oquetambém foi verificado por CHERIAN; SIM (1991) e JIANG etal.(1991). A gordura da gema é composta de aproximadamente 29,7\% defosfolipídios, 63,3\% detriacilgliceróise $4,9 \%$ de colesterol livre. Os fosfolipídios (média de 23,9\% de fosfatidiletanolamina e $69,1 \%$ de fosfatidilcolina) em menor teor no ovo mostram, conseqüentemente, menor percentual de incorporação de EPA e DHA nos tecidos e gema de ovos (LESKANICH; NobLE, 1997).

Analisando o consumo de suplementos ricos em DHA (Tabela 1) nesta pesquisa, a maior eficiência de incorporação de DHA na gema do ovo foi de 85,11\% (OP120), quando as aves tiveram a ingestão média de 123,57 mg de DHA/ave/dia e apresentaram enriquecimento dos ovos com 104,80 mg DHA/gema, decrescendo a eficiência deincorporação para 49,45\% (OP360) e 34,03\% (AM420) de DHA na gema dos ovos. Este decréscimo linear segundo o aumento de consumo de DHA das fontes pode ser expresso pelas equações: OP: $\mathrm{Y}=-8,7443 \mathrm{X}+88,4970, \mathrm{R}^{2}=0,85$ e de $\mathrm{AM}: \mathrm{Y}=$ $7,1074 X+71,7190, \mathrm{R}^{2}=0,79$.

Com níveis de $360 \mathrm{mg}$ (OP360) e $420 \mathrm{mg}$ de DHA (AM420) das fontes OP e AM na ração e as ingestões médias de 380 mg DHA (OP360) e 440,21 mg DHA/ ave/dia (AM420), respectivamente, propiciaram fortificação dos ovos com 218,22 mg (OP) e 175,32 mg/ gema (AM) de PUFAs n-3 (Tabela 5).

Os valores deenriquecimento deovoscomPUFAsn3 estão de acordo com CHERIAN et al. (1996) e CHERIAN; SIM (2003) que reportarama incorporação, respectivamente, de3,8\% e 4,5\% deDHAna gema deovoscom 3,5\% deóleo de savelha na dieta de poedeiras. SURAI; SPARKIS (2001) relataram a fortificação de ovos com $200 \mathrm{mg} /$ ovo de PUFAsn-3. HARGIs etal. (1991) utilizaram 3\% deóleo de peixeeobtiveram acima de $180 \mathrm{mg} /$ ovo deEPA + DHA. SIM (1998) mencionou o uso da semente de linhaça na produção de ovos enriquecidos e o total de PUFAs n-3 variou de $7 \%$ a $12 \%$ dos lipídeos da gema e os teores de ácidos graxos nos ovosem ordem decrescente foramde: $\alpha$-linolênico $>$ DHA $>$ DPA $>$ EPA.

Nesta pesquisa, os resultados evidenciando aumentos significativos, atingindo 3,85\% do total de PUFAs n-3 comparados ao grupo CON, são concordantes com as afirmações dos autores acima ao observarem que através o desenho de projeto experimental, a possibilidade da obtenção de ovos enriquecidos em PUFAs n-3. Entretanto, nas condições experimentais desta pesquisa, a maior média $(218,22 \mathrm{mg}$ / gema para o total dePUFAsn-3) mostrou-se diferente do valor de $600 \mathrm{mg}$ /ovomencionada pelos autores acima(Tabela5).

Ouso de AMnesta pesquisa possibilitou o enriquecimento deovoscom175,32mgdePUFAsn-3/gemaao suplementar poedeiras com 420 mg DHA/100 g ração. Resultados semelhantes foram relatados por ABRIL; BARCLAY (1998) ao utilizarem $300 \mathrm{mg}$ de DHA e $600 \mathrm{mg}$ de DHA/ave/dia de microalga marinha e os ovos enriquecidos apresentaram 172,8 e $243 \mathrm{mg}$ do total de PUFA n-3, respectivamente, e por HERBER;VAN ELSWYK (1996) ao suplementarem a ração de poedeiras com $2,4 \%$ e $4,8 \%$ de microalga marinha Schizochytrium sp. e ovos fortificados mostraram $9,5 \mathrm{mg} / \mathrm{ge} 11,5 \mathrm{mg} / \mathrm{g}$ de gema de PUFAs n-3, respectivamente.

As assertivas apresentadas pelos autores acima, em relação a influencia do suplemento de DHA na ração sobre o aumento dos teores totais de PUFAs n3 na gema, corroboram os resultados desta pesquisa ao ser evidenciada melhora significativa nas relações de n-6:n-3, decrescendo de 17,50 (CON) para 6,36 (AM420) e 3,72 (OP360) (Tabela 3). Da mesma maneira, CHERIAN; SIM (2003) reportaram ouso de 3,5\% deOP e decréscimo significativo na relação deácidos graxos n-6:n-3 de 10:1 para 1:1 nos ovos enriquecidos com n-3. 
Os requerimentos de PUFAs por humanos adultos, segundo Simopoulos etal. (1994), baseando-se nas tabelas "Recommended Dietary Allowance" (RDA) presentes nas decisões do Subcomitê de Nutrição acrescidas ao National ResEARCH COUnCIL (1989), seriam no mínimo de $220 \mathrm{mg}$ de DHA e $220 \mathrm{mg}$ de EPA/ dia. Os ovos resultantes desta pesquisa contemplaram o suprimento das exigências míninas de $85 \%$ e $68 \%$ de DHA com a ingestão de um ovo/dia por humanosadultos, provenientes das dietas com OP360 e AM420, respectivamente (Tabela 5).

\section{CONCLUSÕES}

Ficou constatada a possibilidade de manipulação da composição dosácidos graxos da gordura da gema do ovo favorável ao aumento de PUFAs n-3 a partir de fontes suplementares de ácidos graxos de OP e AMà dieta de poedeiras.

O peso e teor de gordura total da gema não foram influenciados pelas fontes de ácidos graxos na ração.

O aumento dos PUFAs n-3 nas rações contendo OP e AM determinaram resposta linear no aumento de DHA e totais de PUFAs n-3 na gema do ovo, sendo a fonte deOP mais efetiva, proporcionando as melhores médias de 187,91 mg de DHA e 218,22 mg de PUFAs n-3 e da relação de PUFAs n-6:n-3 $(3,72)$.

Os melhores percentuais de incorporação $(85,11 \%)$ de DHA na gema do ovo ocorreram com a suplementação da fonte de $\mathrm{OP}$ à dieta, decrescendo significativamente em relação inversa ao consumo de DHA na ração.

A praticidade dos resultados gerados permite a implementação de estratégias para produção de ovos projetados e enriquecidos em DHA e PUFAs n-3 visando atender a demanda da indústria junto à cadeia de produção avícola.

A ingestão de um ovo/dia atenderia as necessidades diárias de aproximadamente $85 \%$ de DHA no homem adulto, segundo a tabela de exigências da "Recommended Dietary Allowance" (RDA).

A fonte de OP mostrou viabilidade prática para uso extensivoemenriquecimento de ovos comDHA ePUFAs n-3atéolimiteentre2\% a 2,5\%, excetoparausoindustrial, faceàscaracterísticas organolépticas doovoresultantede dietascomestesuplemento.Poroutrolado, ouso dafonte de AM poderá ser estimuladocomincremento da produção de biomassa industrial rica em PUFAs n-3.

\section{Comitê de Bioética}

Projeto de Pesquisa aprovado pelo parecer n ${ }^{\circ} 364$ / 2003 da Comissão de Bioética da Faculdade de Medicina Veterinária e Zootecnia da Universidade de São Paulo.

\section{REFERÊNCIAS}

ABRIL, J.R.; BARCLAY, W.R.; ABRIL, P.G. Safe use of microalgae (DHA GOLD ${ }^{\mathrm{TM}}$ ) in laying hen feed for the production of DHA-enriched eggs. In: SIM, J.S.; NAKAI, S.; GUENTER, W. (Ed.). Egg nutrition and biotechnology. New York: CABI Publishing, 2000. p.197-202.

ABRIL, J.R.; BARCLAY, W. Production of docosahexaenoic acid-enriched poultry eggs and meat using an algae-based feed ingredient. World Review of Nutrition and Dietetics, v.83, p.77-88, 1998.

ASSOCIATION OF OFFICIAL AGRICULTURAL CHEMISTS. AOAC Official Methods of Analysis. 12. ed. Washigton, DC: AOAC, 1970. 1094p.

BARCLAY, W.; ABRIL, R.; ABRIL, P.; WEAVER, C.; ASHFORD, A. Production of docosahexaenoic acid from microalgae and its benefits for use in animal feeds. World Review of Nutrition and Dietetics, v.83, p.6176, 1998.

BLIGH, E.G.; DYER, W.J. A rapid method of total lipid extraction and purification. Canadian Journal of Biochemistry and Physiology, v.37, p.911-917, 1959.

CHEN, P.H.; COMMON, R.H.; NIKOLAICZUK, N.; MACRAE, H.F. Some effects of added dietary fats on the lipid composition of hen's egg yolk. Journal of Food Science, v.30, p.838-845, 1965.

CHERIAN, G.; SIM, J.S. Effect of feeding full fat flax and canola seeds to laying hens on the fatty acid composition of eggs, embryos, and newley hatched chicks. Poultry Science, v.70, n.4, p.917-922, 1991.

CHERIAN, G.; SIM, J.S. Maternal and posthatch dietary polyunsaturated fatty acids alter tissue tocopherol status of chicks. Poultry Science, v.82, n.4, p.681-686, 2003.

CHERIAN, G.; WOLFE, F.H.; SIM, J.S. Feeding dietary oils with tocopherols. Effects on internal qualities of eggs during storage. Journal of Food Science, v.61, n.1, p.15-18, 1996.

COTTERIL, O.J.; GEIGER, G.S. Egg product yield trends from shell eggs. Poultry Science, v.56, n.3, p.1027-1031, 1977.

CRUICKSHANK, E.M. The composition of the egg fat and depot fat of the fowl as affected by the ingestion of large amounts of different fats. The Biochemical Journal, v.28, p.965-977, 1934.

DONALDSON, W.E. Fatty acid interconversion by laying hens. Poultry Science, v.45, n.3, p.473-478, 1966.

ETCHES, R.J. Reproduction in poultry. Guelph: CAB International, 1996. 318p. 
FISHER, H.; LEVEILLE, G.A. Observations on the cholesterol, linoleic and linolenic acid content of eggs as influenced by dietary fats. Journal of Nutrition, v.63, n.1, p.119-129, 1957.

FOLCH, J.; LEES, M.; SLOANE-STANLEY, G.H. A simple method for the isolation and purification of total lipids from animal tissues. Journal of Biological Chemistry, v.226, n.1, p.479-509, 1957.

GALOBART, J.; BARROETA, A.C.; CORTINAS, L.; BAUCELLS, M.D.; CODONY, R. Accumulation of alpha-tocopherol in eggs enriched with omega-3 and omega-6 polyunsaturated fatty acids. Poultry Science, v.81, n.12, p.1873-1876, 2002.

GRIFFIN, H.D. Manipulation of egg yolk cholesterol: a physiologist's view. World's Poultry Science Journal, v.48, p.101-112, 1992.

GRIFFIN, H.D.; PERRY, M.M.; GILBERT, A.B. Yolk formation. In: FREEMAN, B.M. (Ed.). Physiology and biochemistry of the domestic fowl. London: Academic Press, 1984. v.5, p.345-379.

GRIMINGER, P. Lipid metabolism. In: STURKIE, P.D. (Ed.). Avian physiology. 4.ed. New York: Springer, 1986. p.345-358.

GUARDIOLA, F.; CODONY, R.; RAFECAS, M.; BOATELLA, J.; LOPEZ, A. Fatty acid composition and nutritional value of fresh eggs from large-and smallscale farms. Journal of Food Composition and Analysis, v.7, p.171-188, 1994.

HARGIS, P.S.; van ELSWYK, M.E.; HARGIS, B.M. Dietary modification of yolk lipid with menhaden oil. Poultry Science, v.70, n.4, p.874-883, 1991.

HARTMAN, L.; LAGO, R.C.A. Rapid preparation of fatty acid methyl esters from lipids. Laboratory Practice, v.22, p.475-477, 1973.

HERBER, S.M.; van ELSWYK, M.E. Dietary marine algae promotes efficient deposition of n-3 fatty acids for the production of enriched shell eggs. Poultry Science, v.75, n.12, p.1501-1507, 1996.

JIANG, Z.R.; AHN, D.U.; SIM, J.S. Effects of feeding flax and two types of sunflower seeds on fatty acid compositions of yolk lipid classes. Poultry Science, v.70, n.12, p.2467-2475, 1991.

JIANG, Z.; SIM, J.S. Consumption of n-3 polyunsaturated fatty acid enriched eggs and changes in plasma lipids of human subjects. Nutrition, v.9, n.6, p.513-518, 1993.

JIANG, Z.; SIM, J.S. Fatty acid modification of yolk lipids and cholesterol-lowering eggs. In: SIM, J.S.; NAKAI, S (Ed.). Egg uses and processing technologies: new developments. Wallingford, Oxon: CAB International, 1994. p.349-361.
LESKANICH, C.O.; NOBLE, R.C. Manipulation of the n-3 polyunsaturated fatty acid composition of avian eggs and meat. World's Poultry Science Journal, v.53, n.2, p.155-183, 1997.

LI-CHAN, E.C.Y.; POWRIE, W.D.; NAKAI, S. The chemistry of eggs and egg products. In: STADELMAN, W.J.; COTTERILL, O.J. (Eds.). Egg science and technology. 4.ed. New York: Food Products Press, 1995. p.105-175.

MARSHALL, A.C.; KUBENA, K.S.; HINTON, K.R.; HARGIS, P.S.; van ELSWYK, M.E. n-3 fatty acid enriched table eggs: a survey of consumer acceptability. Poultry Science, v.73, n.8, p.1334-1340, 1994a.

MARSHALL, A.C.; SAMS, A.R.; Van ELSWYK, M.E. Oxidative stability and sensory quality of stored eggs from hens fed 1,5\% menhaden oil. Journal of Food Science, v. 59, n. 3, p. 561-563, 1994b.

MORI, A.V. Utilização de óleo de peixe e linhaça na ração como fontes de ácidos graxos poliinsaturados ômega-3 em ovos. 2001. 162f. Tese (Doutorado em Clínica Médica) Faculdade de Medicina Veterinária e Zootecnia, Universidade de São Paulo, São Paulo, 2001.

NABER, E.C.; BIGGERT, M.D. Patterns of lipogenesis in laying hens fed a high fat diet containing safflower oil. Journal of Nutrition, v.119, n.5, p.690-695, 1989.

NATIONAL RESEARCH COUNCIL. U.S. Recommended dietary allowances. RDA. 10.ed. Washington: National Academy Press, 1989. 284p.

NATIONAL RESEARCH COUNCIL. U.S. Nutrient requeriments of poultry. 9.ed. Washigton: National Academy of Sciences, 1994. p.155.

NETTLETON, J.A. Omega-3 fatty acids and health. New York: Thomson Publishing, 1995. 359p.

NEURINGER, M.; CONNOR, W.E.; PETTEN, C.V.; BARSTAD, L. Dietary omega-3 fatty acid deficiency and visual loss in infant rhesus monkeys. Journal of Clinical Investigation, v.73, p.272-276, 1984.

NIELSEN, H. Hen age and fatty acid composition of egg yolk lipid. British Poultry Science, v.39, n.1, p.53-56, 1998.

NOBLE, R.C.; COCCHI, M.; TURCHETTO, E. Egg fat a case for concern? World's Poultry Science Journal, v.46, p.109-118, 1990.

PANKEY, R.D.; STADELMAN, W.J. Effect of dietary fats on some chemical and functional properties of eggs. Journal of Food Science, v.34, p.312-317, 1969.

PHETTEPLACE, H.W.; WATKINS, B.A. Effects of various n-3 lipid sources on fatty acid compositions chickens tissues. Journal of Food Composition and Analysis, v.2, p.104-117, 1989. 
SAS Institute. SAS ${ }^{\circledR} /$ STAT. User's Guide: statistical version. Cary, NC: SAS Institute, 1994.

SCHEUERMANN, G.N.; BELLAVER, C. Estado da arte e a perspectiva para pesquisa futura em nutrição de aves. In: REUNIÃO ANUAL DA SOCIEDADE BRASILEIRA DE ZOOTECNIA, 32., 1995, Brasília, DF. Anais . Brasília, 1995. p.465-473.

SIM, J.S. Designer Eggs and Their Nutritional and Functional Significance. World Review Nutrition Dietetics, v.83, p.89-101, 1998.

SIM, J.S. Designer egg concept: perfecting egg through diet enrichment with w-3 PUFA and cholesterol stability. In: SIM, J.S.; NAKAI, S.; GUENTER, W. (Ed.). Egg nutrition and biotechnology. New York: CABI Publishing, 2000. p.135-150.

SIMOPOULOS, A.P.; KOLETZKO, B.; ANDERSON, R.E.; HORNSTRA, G.; MENSINK, R.P.; WEKSLER, B.B.; HARRIS, W.S.; DE CATERINA, R.; MUGGLI, R.; SPRECHER, H. The 1st Congress of the International Society for the Study of Fatty Acids and Lipids (ISSFAL): fatty acids and lipids from cell biology to human disease. Journal of Lipid Research, v.35, n.1, p.169-173, 1994.

SNEDECOR, G.W.; COCHRAN, W.G. Statistical methods. 6.ed. Ames: University Press, 1967. 593p.

STADELMAN, W.J. Quality identification of shell eggs. In: STADELMAN, W.J.; COTTERILL, O.J. (Ed.). Egg science and technology. 4.ed. New York: Food Products Press, 1995. p.39-66.

STADELMAN, W.J.; PRATT, D.E. Factors influencing composition of the hen's egg. World's Poultry Science Journal, v.45, p.247-266, 1989.

SURAI, P.F.; SPARKS, N.H.C. Designer egg: From improvement of egg composition to functional food. Trends in food Science and Technology, v.12, p.7-16, 2001. van ELSWLYK, M.E.; ASHFORD, A.L.; BARCLAY, W.R.; ABRIL, J.R. Marine microalgae is safe in laying hen rations up to five times the recommended inclusion rate. Poultry Science, v.77, p.42, 1998. Supplement 1.

van ELSWYK, M.E.; HATCH, S.D.; STELLA, G.G.; MAYO, P.K.; KUBENA, K.S. Eggs as a functional food alternative to fish and supplement for the consumption of DHA. In: SIM, J.S.; NAKAI, S.; GUENETER, W. (Ed.). Egg nutrition and biotechnology. New York: CABI Publishing, 2000. p.121-133.

van ELSWYK, M.E. Comparison of n-3 fatty acid sources in laying hen rations for improvement of whole egg nutritional quality: a review. British Journal of Nutrition, v.78, p.S61-S69, 1997. Supplement 1.

WATKINS, B.A.; ELKIN, R.G. Dietary modulation of oleic and stearic acids in egg yolks. Journal of Food Composition and Analysis, v. 5, p. 209-215, 1992.

WHEELER, P.; PETERSON, D.W.; MICHAELS, G.D. Fatty acid distribution in egg yolk as influenced by type and level of dietary fat. Journal of Nutrition, v.69, p.253-260, 1959.

YU, M.M.; SIM, J.S. Biological incorporation of Npolyunsaturated fatty acids into chicken eggs. Poultry Science, v.66, p.195, 1987.

ZEIDLER, G. Eggs vital to human and animal medicine. World Poultry, v.14, n.12, p.33-34, 1998. Disponível em: <http://www.ufrgs.br/lezo/artigos/ texto9.htm>. Acesso em: 17 mar. 2003.

Recebido em 25/9/07

Aceito em 13/2/09 